\title{
Rapid Discrimination of Maggots Utilising ATR-FTIR Spectroscopy
}

Claire L. Pickering ${ }^{a, c}$, James R. Hands ${ }^{a, c}$, Leanne M. Fullwood ${ }^{a}$, Judith A. Smith ${ }^{b}$,

$$
\text { Matthew J. Baker }{ }^{a, c^{*}}
$$

a West CHEM, Department of Pure and Applied Chemistry, University of Strathclyde, 295 Cathedral St, G1 1XL, UK

${ }^{\mathrm{b}}$ School of Forensic \& Investigative Sciences, University of Central Lancashire, Maudland Building, Preston, PR1 2HE, UK

${ }^{\mathrm{C}}$ Centre for Materials Science, Division of Chemistry, School of Forensic \& Investigative Sciences, University of Central Lancashire, JB Firth Building, Preston PR1 2HE, UK

${ }^{*}$ Corresponding Author: matthew.baker@strath.ac.uk 


\section{Abstract}

Entomological evidence is used in forensic investigations to indicate time since death. The species and age of maggots or flies that are present at the scene can be used when estimating how much time has passed since death. Current methods that are used to identify species and developmental stage of larvae and fly samples are highly subjective, costly and often time consuming processes and require the expertise of an entomologist or species identification via DNA analysis. The use of vibrational spectroscopy, as an alternative identification method, would allow for a quicker, cheaper and less subjective technique and would allow entomological evidence to be used more commonly in the forensic process. This proof of principle study shows the potential for using Attenuated Total Reflection-Fourier Transform Infrared spectroscopy (ATR-FTIR) as a rapid tool for differentiating between various species of larvae, such as those commonly found at crime scenes. The proposed regime would provide a rapid and valuable tool resulting in reduced time for both species identification and life cycle determination, particularly in forensic situations.

\section{Keywords}

ATR-FTIR, C. vomitoria, L. sericata, M. domestica, Life cycle, Spectroscopy.

\section{Introduction}

Entomological evidence was first used in death investigations in the $13^{\text {th }}$ century and since then it has developed and advanced to become common place within many forensic investigations. Although the concept has continued to develop, the techniques used for species identification and aging are often very subjective highlighting the need for an alternative technique. Once a body starts to decompose a variety of insects are attracted to the scent of decomposition where certain insects will arrive after different stages of decomposition, and develop through their life cycle in situ allowing an estimate of time providing the samples are still first generation. The species and age of the larvae present on the body can be used to give an indication of post mortem interval (PMI) commonly done using accumulated degree hours/days (ADH/D). ADH is a thermal summation method that takes into account both the time and temperature needed for an insect to develop. The amount of thermal energy required to complete each stage of development is species specific. 
Therefore accurate species identification, age assessment and crime scene temperature measurements are vital to retain accuracy [1-2].

At around 24-48 hours after death the use of PMI calculations begins to break down and entomological evidence, in particular species and age of the samples, becomes more important. Entomologists often look at the size or weight of the larvae present at a crime scene to suggest the developmental stage and give an indication of time since death. However, overcrowding of larvae at the body can cause starvation in some larvae affecting their size and growth in turn affecting the predicted larval stage [3]. Singh et al. looked at how starvation effected the larval development and showed that prolonged periods of starvation experienced in the first two larval stages resulted in all larvae dying while starvation experienced later in the life cycle affected the size of the pupa [4]. Width, as an alternative method to length has been suggested as a less subjective technique to reduce the effect that 'head curling' has on the value PMI value, a factor commonly affecting PMI values calculated when using length. It was concluded that width is comparable with length as a method of age determination and a width value can be simply converted to a length value with $95 \%$ accuracy using a linear model [5]. As metamorphosis causes changes to occur to the larval shape when it develops into an adult fly, these changes can be observed on a molecular level as larvae develop through the life cycle. The rate of development is different for each family of fly but the stages that they develop through are the same, see Figure 1 for general information regarding a typical blowfly lifecycle at $70^{\circ} \mathrm{F}$.

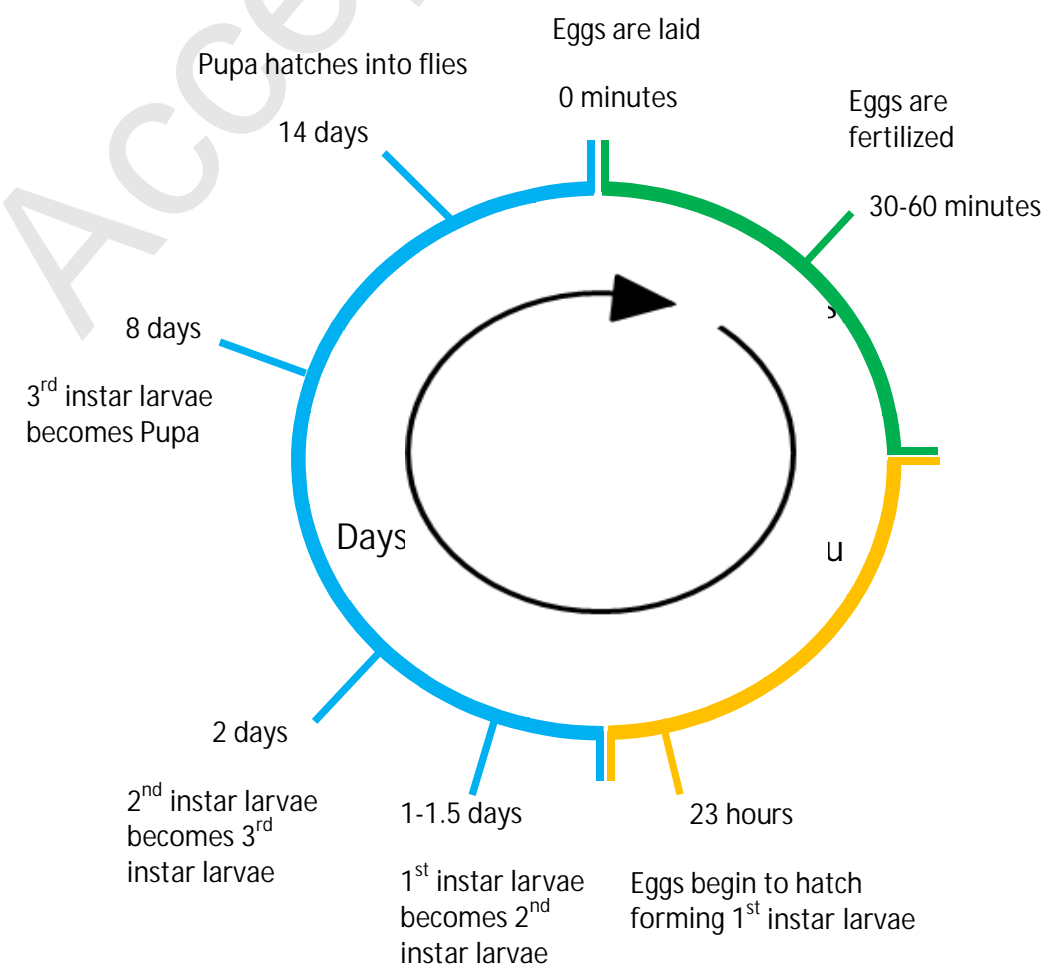


Figure 1. Developmental stages and common timings for a typical blowfly at $70^{\circ} \mathrm{F}$. Adapted from [6]

Quantification of two genes encoding to Actin and Arylphorin Receptor, present in the forensically significant Calliphora vicina, through real-time polymerase chain reaction (PCR) showed the expression of these genes to be age dependent indicating that it is possible to age blow fly larvae using gene expression [7]. Recent studies have highlighted the need for geographically specific data, with regards to development rates, and have shown how important it is to have species specific data to use as a basis rather than to use generalised data. A study of Lucilia sericata which was allowed to develop naturally at different temperatures, carried out in California and Massachusetts, United States of America showed there was a difference in not only their development at different temperatures but also in different geographical locations [8].

As the $3^{\text {rd }}$ stage larvae finishes feeding there is a period of time before the larvae reaches the pupae stage where they will begin to travel away from the body in an attempt to find a safe location where they can pupate. The distance travelled by the $3^{\text {rd }}$ stage larvae can be used as an indication of time since death when species and temperature are known however, the distance travelled will vary greatly depending on the species of larvae and the surface which the larvae have to travel across [9]. Temperature is also considered as it can affect the rate at which the larvae travel, but most importantly the type of scene, whether it is indoor or outdoor, is taken into account as an indoor scene will limit the distance that can be travelled. Colonisation on a fresh carcass is also affected by scene environment, again whether it is an indoor or an outdoor scene, and it has been suggested that it could be up to 24 hours before an indoor carcass is colonised due to blowflies entering indoor environments resulting in a lower number of eggs and less metabolic heat being produced which are both factors that affect the PMI value [10].

As entomological evidence is used in many investigations the way in which the evidence is analysed needs to be accurate ensuring that the evidential information obtained from the samples is of the highest standard and can stand up to scrutiny in 
court. There is a requirement for a new method of species and age identification that can be objective, quick and easy to use but most importantly one that speeds up the identification process.

Vibrational spectroscopies, such as FTIR, are ideal to be used with biological samples as they are label-free [11], rapid, non-destructive techniques that require little sample preparation and are cost effective [12]. There are major 3 modes used for sampling in Infrared spectroscopy; Transmission, Reflectance and Attenuated Total Reflection (ATR) [13]. The spectra collected using IR spectroscopy produces a 'fingerprint' for each sample that is analysed and can be used to characterise a sample. Handheld IR instrumentation makes it the technique very portable and adaptable to use in situ within forensic arenas.

Near Infrared (NIR) spectroscopy has previously been used to determine a number of traits including species, age and if the fly had Wolbachia infection [14-15]. One study looked only at identifying species (Drosophila obscura and Drosophila subobscura) with NIR achieving a $85 \%$ accuracy in wild caught maggots with a slightly higher accuracy in laboratory reared maggots of between 87-92\%.[14] Another study looked at species, age and disease state in laboratory-reared Drosophila melanogaster and Drosophila simulans [15]. Correct speciation of $D$. Melanogaster and D. Simulans achieved accuracies of $94 \%$ and $84 \%$ respectively. The aging study produced accuracies between 66-88\% for flies younger than 9 days with flies aged 9 days or older achieving a accuracy of $91-98 \%$. In this case NIR was also used to identify of the flies were infected with Wolbachia achieving a rate of around $90 \%$ accuracy. The results obtained from these two studies using NIR showed positive results for identification on most occasions, generally achieving over $80 \%$ for each aspect with the accuracy only dropping for samples under nine days old. Attenuated Total Reflection- Fourier Transform Infra-red Spectroscopy (ATRFTIR) has the potential to be a more practical technique that can easily be applied to forensic situations as it provides more detailed spectra, in particular within the fingerprint region, than that of NIR which is vital for identifying both species and life cycle.

This proof of principle project looks at the use of ATR-FTIR spectroscopy as a rapid, objective technique for the identification of maggots. Three species commonly 
encountered at crime scenes have been used, C. vomitoria, $L$. sericata and $M$. domesticus, to enable relevance to forensic situations. In addition, the study analysed the ability of ATR-FTIR to determine the life cycle stage.

\section{Methods and materials}

\section{Genetic Species Confirmation}

A single sample of each of the following species $C$. vomitoria, L. sericata and $M$. domestica were obtained and frozen at $-20^{\circ} \mathrm{C}$. Each sample was defrosted and homogenised using an eppendorf tube and a micropestle. DNA was extracted using the QI Amp system following the manufacturer's protocol (Qiagen Ltd, UK). Each sample was quantified using the Nanodrop 2000 spectrophotometer. Between 1-10 ng total genomic DNA was used to amplify a 524 bp fragment of the Cytochrome Oxidase 1 gene in a $25 \mu \mathrm{L}$ reaction with $1 x$ Reddy mix (1.5 mM MgCl , ABgene) 0.4 $\mu \mathrm{M}$ forward and reverse primers [16] Cycling Parameters - initial denaturation at $94^{\circ} \mathrm{C}$ for 2 mins, followed by 33 cycles of $94^{\circ} \mathrm{C}, 1 \mathrm{~min} ; 54^{\circ} \mathrm{C} 1 \mathrm{~min} ; 72^{\circ} \mathrm{C} 1 \mathrm{~min}$; and a final extension at $72^{\circ} \mathrm{C}$ for 15 mins. The samples were visualised using a $1.2 \%$ agarose gel and sequenced.

The PCR products were purified using Microclean (Web Scientific, Ltd, UK) using the manufacturers' conditions. Sequencing was carried out with BigDye Teminator v 3.1 (Applied Biosystems, USA) in a $10 \mu \mathrm{L}$ reaction containing $0.75 \mu \mathrm{L}$ reaction mix, $1.7 \mu \mathrm{L}$ sequencing buffer, $10 \mathrm{ng}$ PCR product, $3.2 \mathrm{pmol}$ primer, and PCR grade $\mathrm{H}_{2} \mathrm{O}$. Sequencing Parameters: denaturation at $96^{\circ} \mathrm{C} 1 \mathrm{~min}$, followed by 25 cycles of $96^{\circ} \mathrm{C}$ $10 \mathrm{sec} ; 50^{\circ} \mathrm{C} 5 \mathrm{sec} ; 60^{\circ} \mathrm{C} 4 \mathrm{~min}$. Reactions were purified using the sodium acetate, EDTA, glycogen and ethanol precipitation method and run on the 3500 Genetic Analyser using POP- $6^{\mathrm{TM}}$ and the default run parameters (POP- $7^{\mathrm{TM}}$ polymer, $50 \mathrm{~cm}$ array, run module: StdSeq50_POP7, dye set: $Z$. run with an 8 second injection at $1.6 \mathrm{kV}$ and a run time of 96 minutes at $60^{\circ} \mathrm{C}$ and $8.5 \mathrm{kV}$ ). Sequences were analysed using DNA Sequencing Analysis Software v5.4 (Applied Biosystems, USA). The sequences were then compared by a BLAST search of the NCBI database (GenBank). Species identity was confirmed with a $99 \%$ match $(M$. domestica and $L$. sericata) and $100 \%$ match (C. vomitoria) to a voucher sequence for each species. 


\section{Spectral collection}

Spectral collection was carried out at the University of Central Lancashire using a JASCO FTIR-410 Specac ATR single reflection diamond Golden Gate ${ }^{\text {TM }}$ spectrometer in absorption mode using a range of $4000-400 \mathrm{~cm}^{-1}$, at a resolution of $4 \mathrm{~cm}^{-1}$ with 32 co-added scans. Once collection was complete a visual spectral quality test was performed to identify gross error before pre-processing techniques applied to the data set using MATLAB.

\section{Drying study}

A drying study was carried out looking at the affect that the drying time of the sample had on the spectra produced and looked for the optimal time for the sample to dry onto the crystal. It was important for the sample to have an intimate contact with the crystal to ensure the best quality spectra were produced. A sample was applied to the crystal and allowed to dry with spectra being collected a various time points from 0-20 minutes, see Figure 2. It was decided that 15 minutes was the optimum drying time for the samples.

For species identification study using ATR-FTIR

50 of each of the following species $C$. vomitoria, L. sericata and $M$. domestica were obtained and frozen and stored at $-25^{\circ} \mathrm{C}$. Each sample was defrosted before being crushed to a mixture of epidermis and internal matter using an eppendorf tube and a micropestle. A small amount of the internal matter was applied to cover the ATRFTIR crystal. Before the sample was applied to the crystal a background spectrum was acquired and compared to a standard background spectrum to ensure the crystal had been fully cleaned. When the sample was first applied to the crystal, a spectrum was collected before the sample was allowed to dry for 15 minutes before collecting 3 more spectra. For each maggot collecting an initial spectrum along with the three collected after 15 minutes is termed a run. Spectra were collected over the range of $4000-650 \mathrm{~cm}^{-1}$ with particular interest in the fingerprint region of 900-1800 
$\mathrm{cm}^{-1}$. Virkon (purchased from Antec Int., Suffolk, UK) and absolute ethanol were used to clean the ATR-FTIR crystal in between runs.

For life cycle study using ATR-FTIR

C. vomitoria was chosen for the life cycle study where the sample set consisted of collections from 17 time points within the developmental stages shown in Table 1.

Table 1 Developmental stage and ADH values

\begin{tabular}{|c|c|}
\hline Life cycle stage & Number of time points per life cycle stage \\
\hline Egg & 2 \\
\hline $1^{\text {st }}$ Larval stage & 3 \\
\hline $2^{\text {nd }}$ Larval stage & 3 \\
\hline $2^{\text {nd }} / 3^{\text {rd }}$ Larval stage border & 1 \\
\hline $3^{\text {rd }}$ Larval stage & 2 \\
\hline $3^{\text {rd }}$ Larval stage (Post feeding) & 3 \\
\hline Pupa & 2 \\
\hline Fly & 1 \\
\hline
\end{tabular}

Six samples from each time point in the life cycle of $C$. vomitoria were used and spectra were collected. Larval stages were confirmed by observation of the number of slits in the posterior spiracle which increases with each larval molt [17]. The samples were defrosted before they were crushed to a mixture of epidermis and internal matter using an eppendorf tube and a micropestle. A small amount of the internal matter was spread onto the ATR-FTIR crystal. Before the sample was applied to the crystal a background spectrum (for atmospheric correction) was taken and compared to a standard background spectrum to ensure the crystal had been fully cleaned. When the sample was first applied to the crystal, a spectrum was collected before the sample was allowed to dry for 15 minutes before collecting 3 more spectra. For each maggot collecting an initial spectrum along with the three collected after 15 minutes is termed a run. Spectra were collected over the range of $4000-650 \mathrm{~cm}^{-1}$ with particular interest in the fingerprint region of $900-1800 \mathrm{~cm}^{-1}$. Virkon (purchased from Antec Int., Suffolk, UK) and absolute ethanol were used to clean the ATR-FTIR crystal in between runs. 
The $C$. vomitoria samples bought from a tackle shop were reared to adulthood at $22^{\circ} \mathrm{C}$ on pork mince at the University of Central Lancashire (UCLan).

\section{Pre-processing and multivariate analysis}

For both the species identification and life cycle studies the same approach was used to pre-process the raw data before analysing it using multivariate analysis. The three spectra collected after 15 minutes were averaged. In the species identification study we performed a visual examination of the spectral quality, for gross spectral error, and removed the identified outliers that were detached from the main body of the spectra to improve the quality of the data set. In both studies the data was noise reduced using 30 principal components, then vector normalised (standard normal variate) and mean centred. Spectral pre-processing was applied to reduce unwanted background information such as offsets and sloped baselines that are due to nonbiochemical information. The species identification study was analysed using Principal Component - Discriminant Function Analysis (PC-DFA) and Support Vector Machine (SVM) while the life cycle study was analysed using Principal Component Analysis (PCA).

There are two main types of multivariate analysis which are supervised and unsupervised. Multivariate analysis is the analysis of multiple variables and can be thought of as pattern recognition algorithms. PC-DFA and SVM are examples of supervised forms of multivariate analysis where the algorithm is given the group numbers and asked to discover similarities within groups and difference between groups. To ensure that the model is robust a blind test set has been created. While SVM is an effective pattern recognition algorithm than PC-DFA no visual representation is produced, whereas PC-DFA creates a scores plot and a loadings plot to allow for easy visualisation of the information [18]. SVM works by putting a hyper plane through the data to separate it out. This hyper plane is multidimensional, making it very difficult to visualise, to allow the best separation of the data. For PCDFA each of the three species were split up into a two thirds training set, used to train the mode, with the remaining one third left to be projected into the model as a blind test. PCA is an example of an unsupervised method of analysis and looks at 
the whole spectral data set to look for natural patterns within the data set and provide information based upon the variance in the dataset.

Sensitivity and specificity values were calculated and used to measure the discrimination of the model. Sensitivity is a measure of the ability to successfully identify the species while specificity measures the ability of the model to not misclassify the species [19]. The sensitivity and specificity values were calculated using equations where equation 1 calculates sensitivity while equation 2 calculates specificity.

\section{Equation for sensitivity}

Sensitivity $=\frac{\text { True Positives }}{\text { True Positives }+F a \text { Ise Negatives }}$

Equation for specificity

Specificity $=\frac{\text { True Positives }}{\text { True Negatives +False Pogitives }}$

Definitions: [12]

True Positives- Number of spectra where samples were correctly identified as their species or life cycle stage.

True Negatives- Number of spectra where samples have been correctly identified as not their species or life cycle stage.

False Positives- Number of spectra where the model has incorrectly identified the samples as a member of a species or life cycle stage which they are not.

False Negatives- Number of spectra where the model has incorrectly identified samples as not a member of their species or life cycle stage.

For both the species identification and the life cycle studies pre-processing and multivariate analysis was carried out on the raw data in MATLAB version 7.11.0 (R2010b) (The MathWorks, Inc., USA) using in-house written software.

\section{$\underline{\text { Results and discussion }}$}




\section{Drying study}

Before any spectra were collected for the species identification study a small drying study was carried out to assess the time required for the samples to dry to a point where an intimate contact was created between the sample and the crystal. The drying study showed that 15 minutes was the optimal drying time to create an intimate contact and was the point where the spectra was considered stable.

This study showed that after 15 minutes the spectra showed no changes and that spreading the innards across the ATR crystal provided the optimum conditions for spectral collection. After 15 minutes the $\mathrm{OH}$ peak, in the region of $3000-3600 \mathrm{~cm}^{-1}$, had significantly dropped in intensity indicating that the samples was dry and had intimate contact with the crystal. 


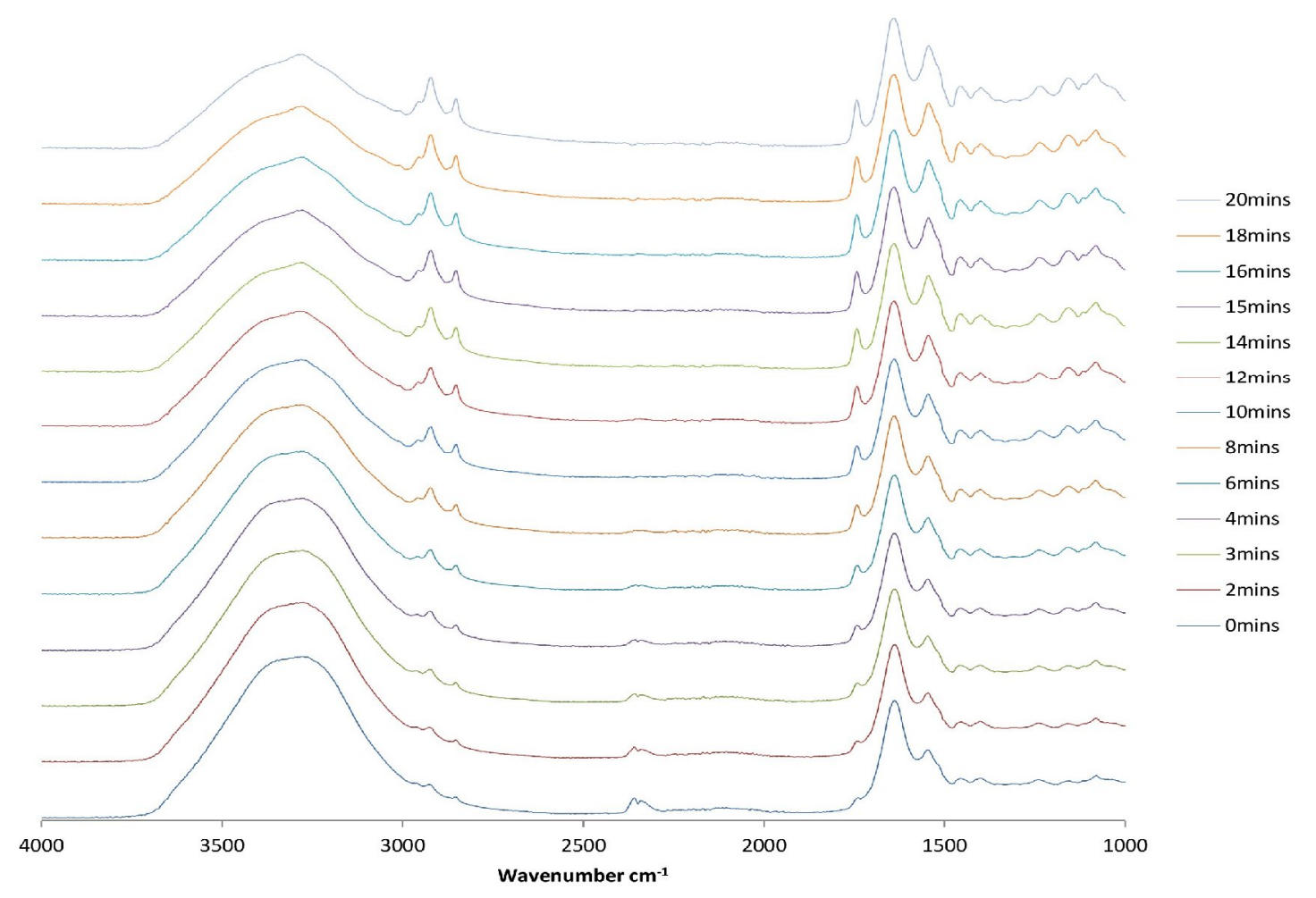

Figure 2 Overlay of the raw spectra collected from 0 to 20 minutes. Spectra have been offset for ease of visualisation.

\section{Species identification study}

Principal Component -Discriminant Function Analysis (PC-DFA) Model

Following pre-processing principal component discrimination function analysis (PCDFA) was performed on the spectra from C. vomitoria $(n=37)$ M. domestica $(n=43)$ and L. sericata $(n=50)$. Sensitivity and specificity values were calculated for each species. C. vomitoria had the best overall values with a sensitivity of $92.86 \%$ and a specificity of $93.10 \%, M$. domestica achieved a sensitivity of $82.35 \%$ and a specificity of $84.62 \%$. L. sericata achieved the best sensitivity value of $100 \%$ but only achieved a specificity value of $48.39 \%$. All values are presented in Table 3 and are supported by Figure 3 which shows the scores plot with $95 \%$ error ellipses. 


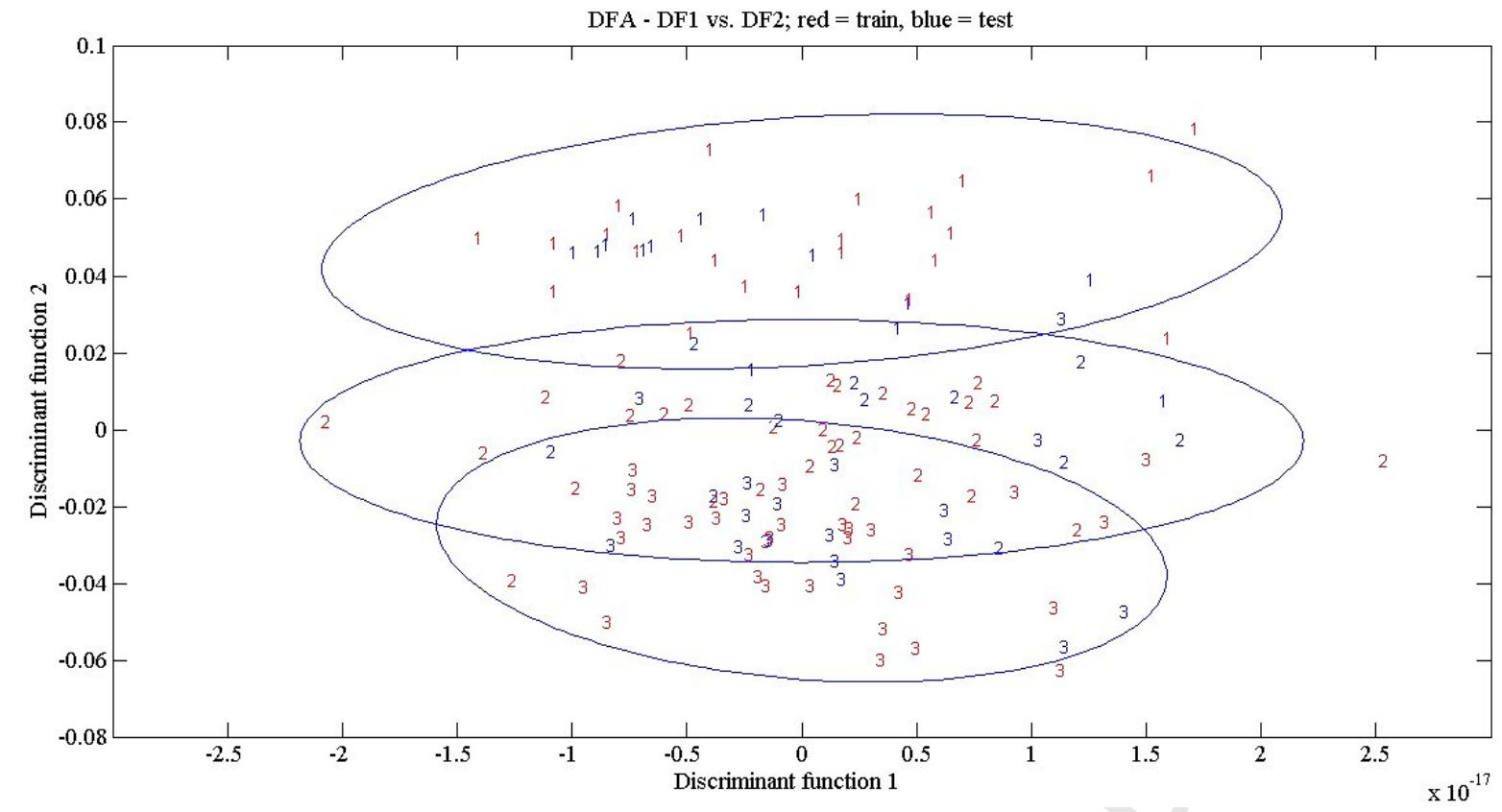

Figure 3 Shows DFA 1 plotted against DFA 2 where 1 represents $C$. vomitoria, 2 represents $M$. domestica and 3 represents $L$. sericata with the red representing the training set and the blue representing the test set. DFA 2 shows the separation between $C$. vomitoria and $L$. sericata whereas DFA 1 shows no real separation.

Table 3 Sensitivity and specificity values obtained from species identification study.

\begin{tabular}{|l|l|l|}
\hline Species & Sensitivity (\%) & Specificity (\%) \\
\hline M. domestica & 82.35 & 84.62 \\
\hline C. vomitoria & 92.86 & 93.10 \\
\hline L. sericata & 100 & 48.39 \\
\hline
\end{tabular}

From the known phylogenetic information relating to the three species, $C$. vomitoria and L. sericata are more closely related. The DFA plot shows the relationship between all three species, from phenotypic information probed by ATR-FTIR, but with the closest relationship being between $M$. domestica and $L$. sericata. This may be explained by genetic variations from different genus, based on the fact that $C$. vomitoria and $L$. sericata belong to the same family but belong to different genus [20]. DF2 shows the separation between C. vomitoria and L. sericata, produced the loadings plot represented in Figure 4 suggesting that the separation is mainly down 
to the $\mathrm{C}=\mathrm{O}$ stretch at $1741 \mathrm{~cm}^{-1}$ and the Amide I at $1637 \mathrm{~cm}^{-1}$ and Amide II at $1513 \mathrm{~cm}^{-}$

${ }^{1}$. All tentative peak assignments for DF2 are represented in Table 4.

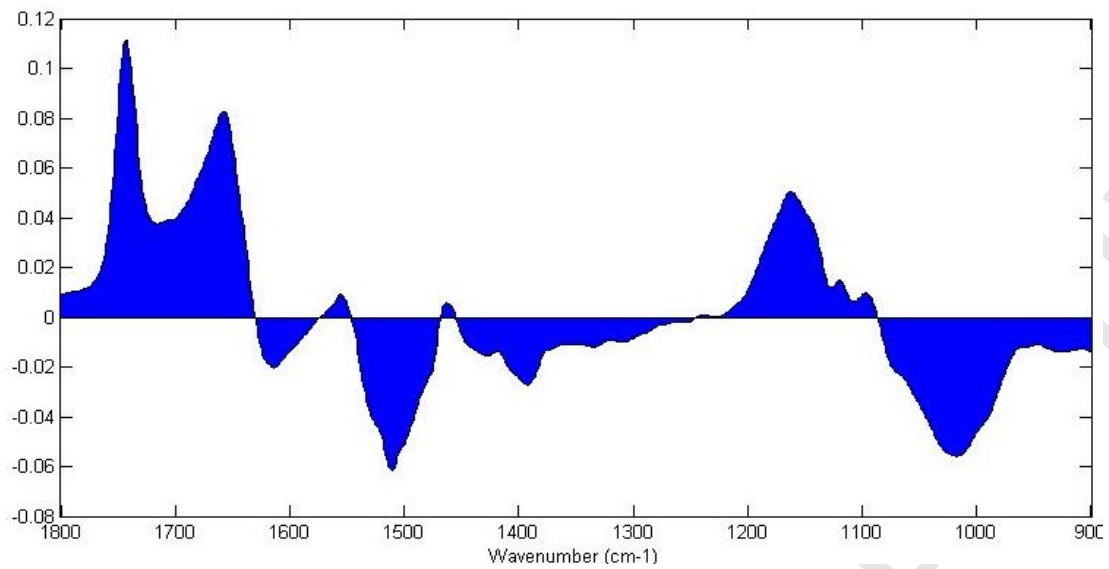

Figure 4 DF2 loadings plot showing peaks $1741 \mathrm{~cm}^{-1}, 1637 \mathrm{~cm}^{-1}$ and $1513 \mathrm{~cm}^{-1}$

Table 4 Major peaks and tentative bio molecular assignments of DF2 (bio molecular peak assignments taken from [21, 12])

\begin{tabular}{|c|c|c|}
\hline Loadings & Wavenumber $\left(\mathrm{cm}^{-1}\right)$ & Proposed bio molecular assignment \\
\hline+ ve & 1741 & $\mathrm{C}=\mathrm{O}$ stretch (symmetric), fatty acids, lipids \\
\hline$+\mathrm{ve}$ & 1637 & $\begin{array}{l}\text { Amide I (Beta- sheet structures) v C=O (80\%), v C-N (10\%), } \delta \\
\qquad \mathrm{N}-\mathrm{H}(10 \%)\end{array}$ \\
\hline$+\mathrm{ve}$ & 1172 & $\begin{array}{l}\mathrm{C}-\mathrm{O}, \mathrm{C}-\mathrm{C} \text { stretch, } \mathrm{C}-\mathrm{O}-\mathrm{H}, \mathrm{C}-\mathrm{O}-\mathrm{C} \text { deformation of carbohydrates or } \\
\mathrm{C}-\mathrm{OH} \text { stretch of serine, threonine, tyrosine in cell proteins }\end{array}$ \\
\hline -ve & 1619 & $\begin{array}{l}\text { Amide I ( } \beta \text { sheet structures) v } \mathrm{C}=\mathrm{O}(80 \%), v \mathrm{C}-\mathrm{N}(10 \%), \delta \mathrm{N}-\mathrm{H} \\
\qquad(10 \%)\end{array}$ \\
\hline -ve & 1513 & Amide II ( $\beta$ sheet structures) $\delta \mathrm{N}-\mathrm{H}(60 \%), v \mathrm{C}-\mathrm{N}(40 \%)$ \\
\hline -ve & 1394 & $\mathrm{CH} 3$ deformation, lipids \\
\hline -ve & 1018 & C-O stretch of glucose \\
\hline
\end{tabular}

\section{SVM results}

SVM was able to achieve superior sensitivity and specificity values compared with those produced using PC-DFA. With SVM, L. sericata achieved the best result with $100 \%$ both sensitivity and specificity. M. domestica was able to achieve a sensitivity of $100 \%$ and a specificity of $96.15 \%$ while C. vomitoria achieved a sensitivity of 
$85.71 \%$ and a specificity of $96.55 \%$, see table 5 . The SVM model produced a cross validation accuracy of $86.21 \%$ with a cost value of 64 and a gamma value of 16 .

Table 5 Table of sensitivity and specificity values created using SVM

\begin{tabular}{|l|l|l|}
\hline Species & Sensitivity (\%) & Specificity (\%) \\
\hline M. domestica & 100 & 96.15 \\
\hline C. vomitoria & 85.71 & 96.55 \\
\hline L. sericata & 100 & 100 \\
\hline
\end{tabular}

\section{Life cycle study}

PCA was performed on the whole data set but showed a poor level of overall discrimination between the different stages. PCA was used to differentiate between two different developmental stages to see if discrimination could be achieved. Figure 5 shows the splitting of two stages of the life cycle data where the L2 data is represented by the blue dots and the L3 data is represented by the green. Figure 5 shows that the best separation is by PC2. If an unknown sample was projected into the model there is a good chance that it would be correctly classified as PCA looks at natural variations in the samples. Classification of L2 and L3 larvae is particularly significant as the $2^{\text {nd }}$ and $3^{\text {rd }}$ larval stages usually occur between 1-8 days after the body death, and account for one of the largest time periods in a typical blowfly lifecycle. 

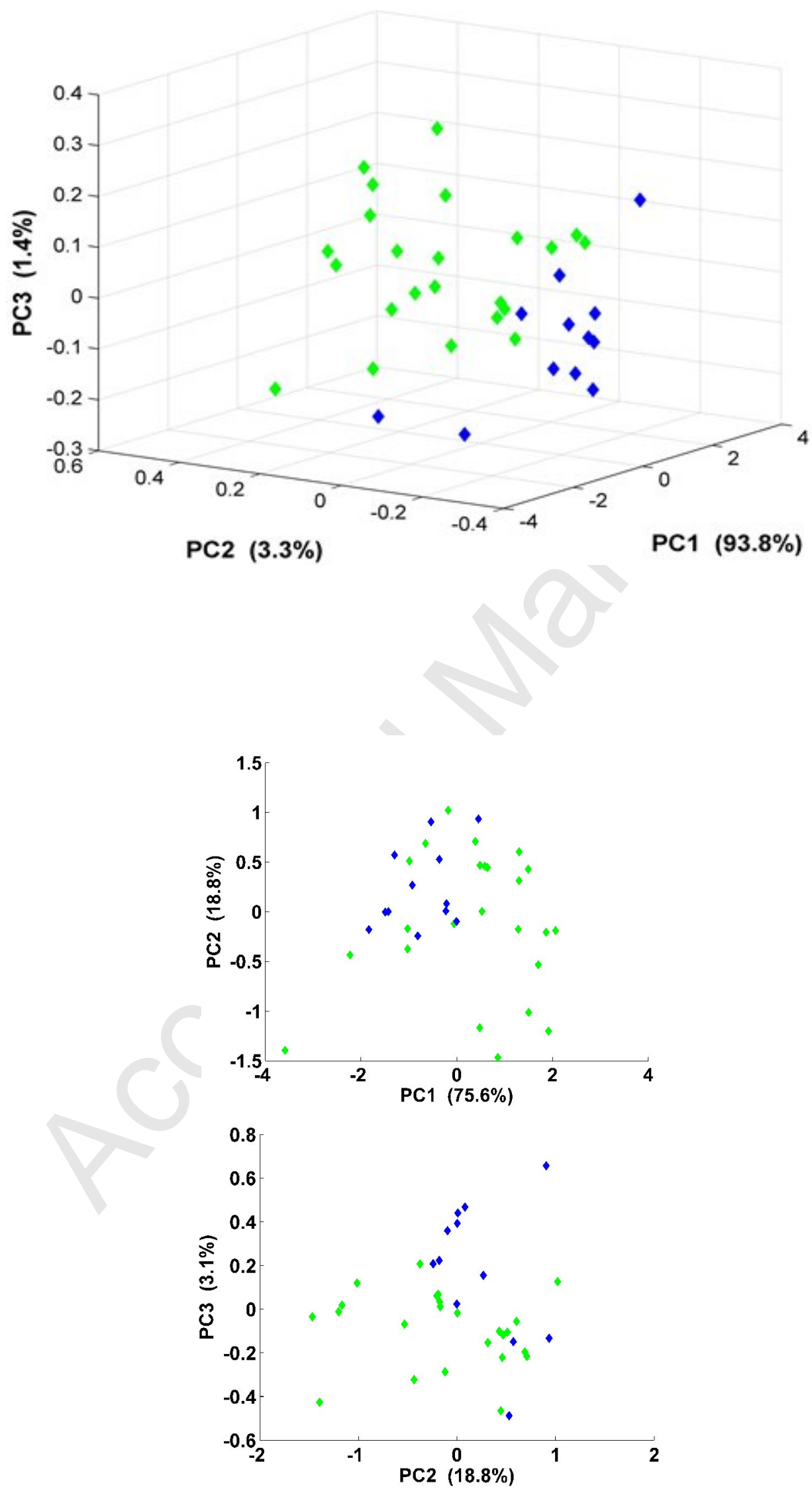


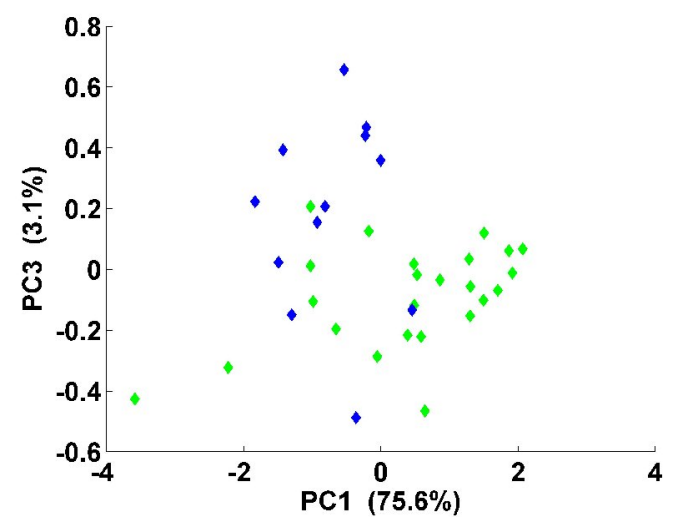

Figure 5 3D-PCA scatter plots of L2 (blue) and L3 (green) samples in the life cycle study.

From figure 5 PC 2 shows the separation along the $x$ axis and produced the loadings plot represented in Figure 6 suggesting that the separation is due to the amide I of proteins at $+1679 \mathrm{~cm}^{-1}$ and the amide II of proteins at $+1561 \mathrm{~cm}^{-1}$ in the positive region and the amide I of proteins at $-1614 \mathrm{~cm}^{-1}$ in the negative region. All tentative peak assignments for PC2 can be seen in table 6 .

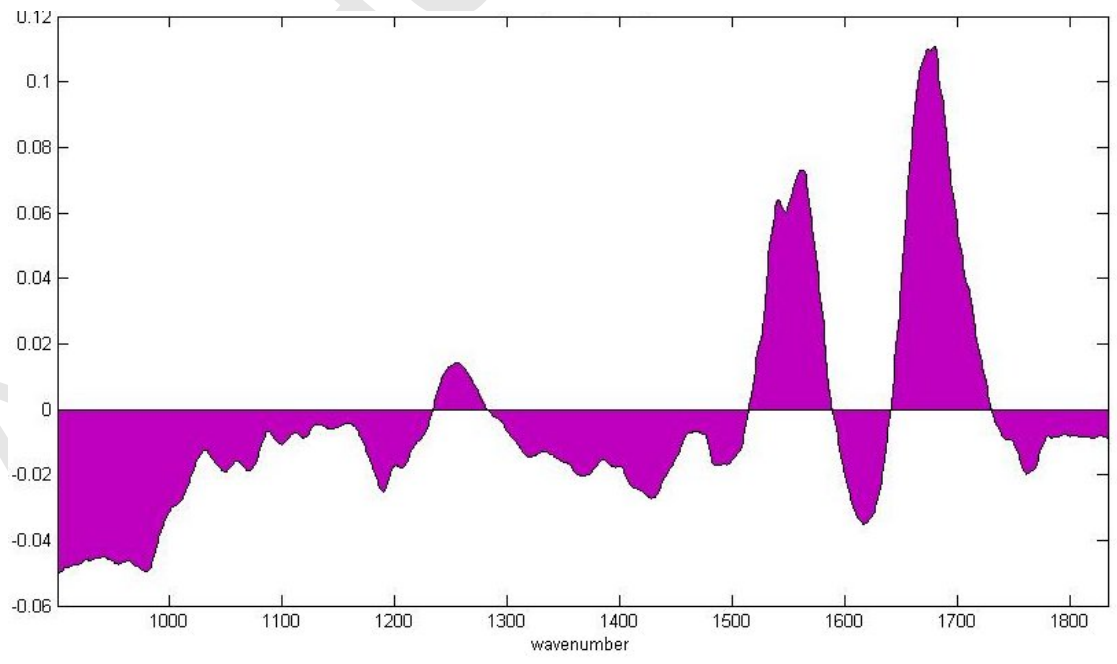

Figure 6 Loadings plot produced by PC2 
Table 6 Major peaks and tentative bio molecular assignments of PC2 (bio molecular peak assignments taken from [21-22])

\begin{tabular}{|c|c|c|}
\hline Loadings & Wavenumber $\left(\mathrm{cm}^{-1}\right)$ & Proposed bio molecular assignment \\
\hline$+\mathrm{ve}$ & 1679 & $\begin{array}{c}\text { Amide I ( } \beta \text { sheet structures) } \vee \mathrm{C}=\mathrm{O}(80 \%), \vee \mathrm{C}-\mathrm{N}(10 \%), \delta \mathrm{N}-\mathrm{H} \\
\qquad(10 \%)\end{array}$ \\
\hline$+\mathrm{ve}$ & 1561 & Amide II ( $\beta$ sheet structures) $\delta \mathrm{N}-\mathrm{H}(60 \%), \mathrm{v} \mathrm{C}-\mathrm{N}(40 \%)$ \\
\hline$+\mathrm{ve}$ & 1261 & $\begin{array}{l}\text { Amide III ( } \mathrm{NH} \text { bend }(55 \%) \text {, CC stretch }(19 \%), \mathrm{CN} \text { stretch }(15 \%) \text {, } \\
\text { CO bend }(11 \%)) .\end{array}$ \\
\hline -ve & 1765 & C-O stretch of carbonic acid \\
\hline -ve & 1614 & $\begin{array}{l}\text { Amide I ( } \beta \text { sheet structures) } \vee \mathrm{C}=\mathrm{O}(80 \%), \vee \mathrm{C}-\mathrm{N}(10 \%), \delta \mathrm{N}-\mathrm{H} \\
\qquad(10 \%)\end{array}$ \\
\hline -ve & 1428 & $\mathrm{CH} 3$ antisymmetric bend \\
\hline -ve & 1192 & $\begin{array}{l}\mathrm{C}-\mathrm{O}, \mathrm{C}-\mathrm{C} \text { stretch, } \mathrm{C}-\mathrm{O}-\mathrm{H}, \mathrm{C}-\mathrm{O}-\mathrm{C} \text { deformation of carbohydrates } \\
\text { or } \mathrm{C}-\mathrm{OH} \text { stretch of serine, threonine, tyrosine in cell proteins }\end{array}$ \\
\hline
\end{tabular}

\section{Conclusions}

The results of the two studies have shown, for the first time that a combination of ATR-FTIR and pre-processing techniques can be used to correctly classify species and life cycle stage. The species identification study showed that a combination of SVM and ATR-FTIR was able to produce the best results producing sensitivities and specificities as high as $100 \%$ for both. The loadings from PC2 show that the separation between these the $2^{\text {nd }}$ and $3^{\text {rd }}$ larval stages is mainly due to differences in Amide I and II present in proteins.

The proposed method of identification and aging, using ATR-FITR spectroscopy and pre-processing, would enhance the use of entomological evidence in forensic arenas with the overall end application of the technique being to identify both species and life cycle stage simultaneously.

\section{$\underline{\text { References }}$}

[1]- J Amendt et al., Best practice in forensic entomology-standards and guidelines, International Journal of Legal Medicine, 121 (2007) 90-104. 
[2]- C. Ames et al., Low temperature episodes in development of blowflies: implications for postmortem interval estimation, Medical and Veterinary Entomology, 17

178-186.

[3]- S. Ireland and B. Turner, The effects of larval crowding and food type on the size and development of the blowfly, Calliphora Vomitoria, Forensic Science International, 159 (2006) 175-181. [4]- D. Singh and M Bala, The effect of starvation on the larval behaviour of two forensically important species of blow flies (Diptera: Calliphoridae), Forensic Science International 193 (2009) 118-121.

[5]- D. M Day and J.F Wallman, Width as an alternative measurement to length for post-mortem interval estimations using Calliphora augur (Diptera: Calliphoridae) larvae, Forensic Science International 159 (2006) 158-167.

[6]-http://www.nlm.nih.gov/visibleproofs/galleries/technologies/blowfly.html-Date accessed 21/07/14.

[7]- P. Boehme, P. Spahn, J. Amendt and R. Zehner, Differential gene expression during metamorphosis: a promising approach for age estimation of forensically important Calliphora vicina pupae (Diptera: Calliphoridae), International Journal of Legal Medicine 127 (2013) 243-249.

[8]- M.B Gallagher, S. Sandhu and R. Kimsey, Variation in developmental time for geographically distinct populations of the common green bottle fly, Lucilia sericata (Meigen), Journal of Forensic Science 55(2) (2010) 438-42.

[9]- S. Arnott and B. Turner, Post-feeding larval behaviour in the blowfly, Calliphora vicina: Effects on post-mortem interval estimates, Forensic Science International 177 (2008) 162-167. [10]- S. Reibe and B Maeda, How promptly do blowflies colonise fresh carcasses? A study comparing indoor with outdoor locations, Forensic Science International 195 (2010) 52-57.

[11]- Baker et al., Using Fourier transform IR spectroscopy to analyse biological materials, Nature Protocols, 9 (2014) 1771-1791.

[12]- J.R Hands et al., Investigating the rapid diagnosis of gliomas from serum samples using infrared spectroscopy and cytokine and angiogenesis factors, Analytical and Bioanalytical Chemistry 405 (2013) 7347-7355. 
[13]- Konrad M. Dorling and Matthew J. Baker, Highlighting attenuated total reflection Fourier transform infrared spectroscopy for rapid serum analysis, Trends in Biotechnology 31 (2013) 327-328.

[14]- S. Fischnaller F. E Dowell, A Lusser, B.C Schlick-Stiener and F. M Steiner, Non-destructive species identification of Drosophila obscura and D. subobscura (Diptera) using near-infrared spectroscopy, Fly 6:4 (2012) 284-289.

[15]- W. C Aw, F. E Dowell and J. W. O Ballard, Using Near-Infrared Spectroscopy to Resolve the Species, Gender, Age, and the Presence of Wolbachia Infection in Laboratory-Reared Drosophila, G3 (Bethesda) 2 (2012) 1057-65.

[16]- J. D Wells and F. A. H Sperling, 'DNA-based identification of forensically important Chrysomyinae (Diptera: Calliphoridae)' Forensic Science International 120 (2001)

110-115.

[17]- T. Klong-klaew et al., Observations on morphology of immature Lucilia porphyrina (Diptera: Calliphoridae), a fly species of forensic importance. Parasitology Research 111 (2012) 1965-1975.

[18]- M.J. Baker et al., ToF-SIMS PC-DFA analysis of prostate cancer cell lines, Applied Surface Science 255 (2008) 1084-1087.

[19]- A. J. S Mclntosh et al., Spectroscopic analysis of bacterial biological warfare simulants and the effects of environmental conditioning on a bacterial spectrum, Analytical and Bioanalytical Chemistry 404 (2012) 2307-2315.

[20]- S. N Kutty, T. Pape, A. Pont, B. M Wiegmann and R. Meier, The Muscoidea (Diptera: Calyptratae) are paraphyletic: Evidence from four mitochondrial and four nuclear genes, Molecular Phylogenetics and Evolution 49 (2008) 639-652.

[21]- J.R Hands et al., Attenuated Total Reflection Fourier Transform Infrared (ATRFTIR) Spectral Discrimination of Brain Tumour Severity from Serum Samples, Journal of Biophotonics, 7 (2014) 189-199.

[22]- M. J Baker et al., Investigating FTIR based histopathology for the diagnosis of prostate cancer, Journal of Biophotonics, 2(2009) 104-113. 


\section{Acknowledgements}

The authors would like to acknowledge $\operatorname{Dr} A$. Henderson from the University of Manchester for providing the MATLAB pre-processing codes used in this research. 